Document downloaded from:

http://hdl.handle.net/10251/159847

This paper must be cited as:

Balbastre Tejedor, JV.; Nuño Fernández, L. (2019). Modelling the propagation of electromagnetic waves across complex metamaterials in closed structures. Journal of Computational and Applied Mathematics. (352):40-49.

https://doi.org/10.1016/j.cam.2018.11.004

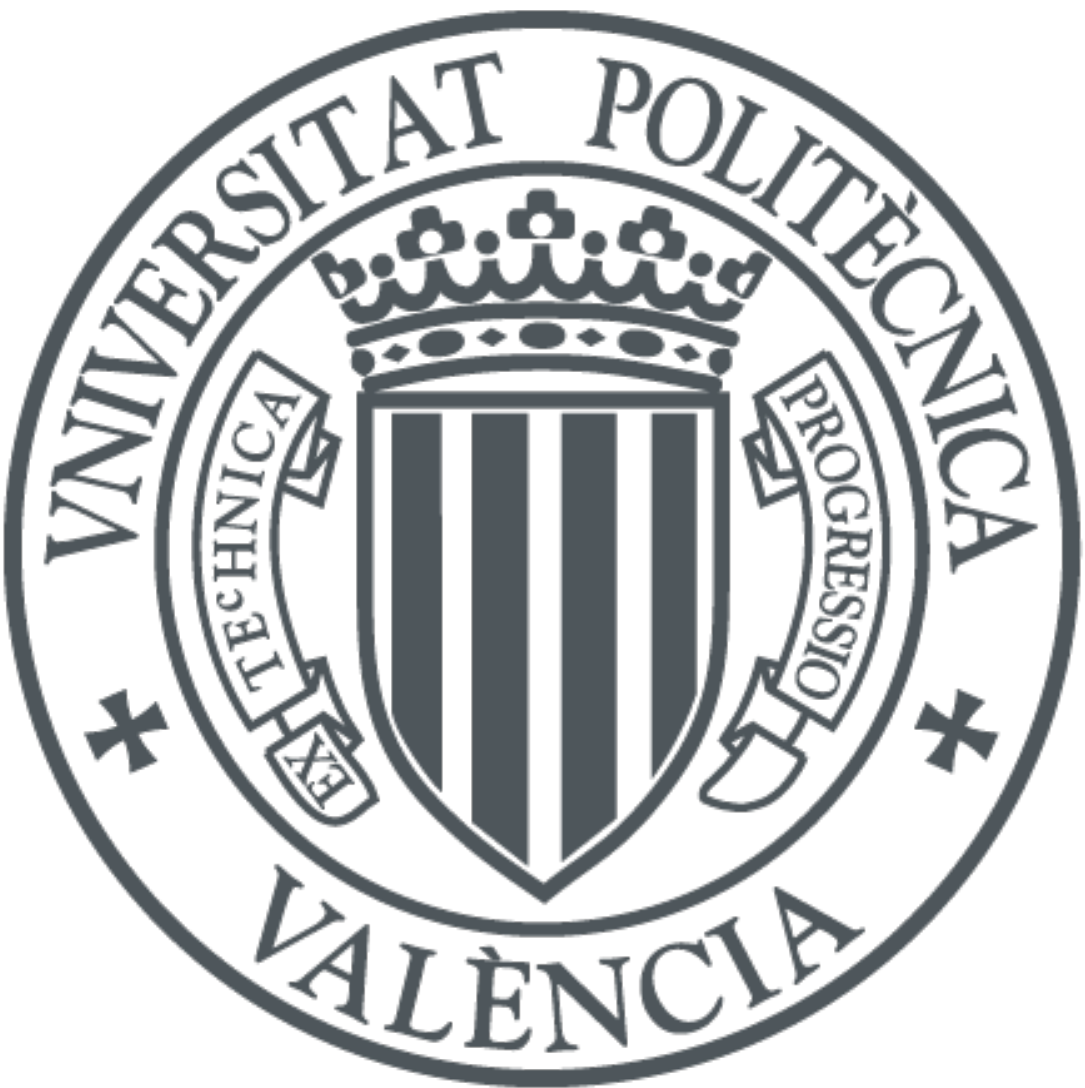

The final publication is available at

https://doi.org/10.1016/j.cam.2018.11.004

Copyright Elsevier

Additional Information 


\title{
Modelling the Propagation of Electromagnetic Waves Across Complex Metamaterials in Closed Structures.
}

\author{
J. V. Balbastre ${ }^{\mathrm{a}, *}$, L. Nuño ${ }^{\mathrm{a}}$ \\ ${ }^{a}$ Instituto ITACA, Universitat Politècnica de València, Valencia, Spain
}

\begin{abstract}
Metamaterials are currently one of the most popular fields in microwave technology because their particular electromagnetic properties lead to a plenty of very relevant applications, both military and civilian. Additionally, the analysis and design of microwave components based on this kind of materials is one of the more challenging problems found by the applied electromagnetism community due to the complexity introduced in the mathematical formulation by their constitutive relationships. The most general case of metamaterial is the bi-anisotropic one, where both the electric field and the electric induction simultaneously depend on the magnetic field and the magnetic induction. In this paper, we present a new and powerful Finite Element Method scheme valid for the analysis of the most general waveguides, filled with lossy bi-anisotropic linear materials. Edge elements have been used in order to prevent the appearance of spurious solutions and the final eigensystems are very sparse, thus allowing a great memory and computing time saving.
\end{abstract}

\section{Introduction}

Gyrotropic magnetised ferrites have been used in microwave engineering for years, because their non-reciprocal behaviour make them very useful in the design of many microwave devices, like isolators, polarizators or circulators. This kind of microwave passive components has been studied in classical textbooks [1,2], as well as in many research papers [3-5]. Materials with anisotropic permittivity and permeability have also been used in Microwave Monolithic Integrated Circuits (MMIC) chip structures [6, 7]. General anisotropic linear materials are characterized by the contitutive relationships

$$
\begin{aligned}
\vec{D} & =\varepsilon_{0} \hat{\varepsilon}_{r} \vec{E}, \\
\vec{B} & =\mu_{0} \hat{\mu}_{r} \vec{H},
\end{aligned}
$$

where $\varepsilon_{0}$ and $\mu_{0}$ are, respectively, the free-space permittivity and permeability and $\hat{\varepsilon}_{r}, \hat{\mu}_{r}$ arbitrary complex tensors. The analysis of waveguides filled with this kind of materials is not evident. So, numerical methods, like the Finite Element Method (FEM), must be used. However, the application of the FEM to electromagnetic problems involving anisotropic materials is not a straightforward task. A considerable effort has been carried out during the last years to find a formulation of FEM valid for the most general waveguide problem. Different solutions for anisotropic waveguides, both involving scalar [8] or vector [9-11] interpolating functions, have been developed and their behavior has been satisfactorily tested in the last years.

More recently, a new kind of materials, electromagnetically active, have been considered for its application in microwave and infrared regions: the chiral materials, a particular case of bi-isotropic materials for which the constitutive relationships take the form

$$
\begin{aligned}
\vec{D} & =\varepsilon \vec{E}-j \zeta_{c} \mu \vec{H}, \\
\vec{B} & =\mu \vec{H}+j \zeta_{c} \mu \vec{E},
\end{aligned}
$$

\footnotetext{
${ }^{*}$ Corresponding author

Email addresses: jbalbast@itaca.upv.es (J. V. Balbastre), lnuno@dcom.upv.es (L. Nuño)
} 
where $\varepsilon$ and $\mu$ are, in general, complex scalars, $\zeta_{c}$ is a real scalar, known as chirality admittance, and $j=\sqrt{-1}$, as it is usual in electrical and electronic engineering.

In [12], Lindell et al. present a detailed historical survey of the theoretical study of chiral materials. In the same reference, as well as in [13], the propagation in waveguides filled with homogeneous chiral materials (chirowaveguides) is studied.

Gyrotropic chirowaveguides appear as a natural extension of chirowaveguides. In this case, the electromagnetic field satisfies

$$
\begin{aligned}
\vec{D} & =\hat{\varepsilon} \vec{E}-j \zeta_{c} \hat{\mu} \vec{H}, \\
\vec{B} & =\hat{\mu} \vec{H}+j \zeta_{c} \hat{\mu} \vec{E}
\end{aligned}
$$

where

$$
\begin{aligned}
& \hat{\varepsilon}=\left[\begin{array}{ccc}
\varepsilon_{1} & -j g & 0 \\
j g & \varepsilon_{1} & 0 \\
0 & 0 & \varepsilon_{2}
\end{array}\right] \varepsilon_{0}, \\
& \hat{\mu}=\left[\begin{array}{ccc}
\mu_{1} & -j \kappa & 0 \\
j \kappa & \mu_{1} & 0 \\
0 & 0 & \mu_{2}
\end{array}\right] \mu_{0},
\end{aligned}
$$

$g$ and $\kappa$ being the parameters describing the transverse anisotropy of the materials. A study of this type of waveguides can be found in $[14,15]$.

Even more complicated cases, like waveguides loaded with Faraday ferrites, for which

$$
\begin{aligned}
\vec{D} & =\hat{\varepsilon} \vec{E}-j \zeta_{c} \hat{\mu} \vec{H}+\zeta_{c}^{2} \hat{\mu} \vec{E}, \\
\vec{B} & =\hat{\mu} \vec{H}+j \zeta_{c} \hat{\mu} \vec{E},
\end{aligned}
$$

have been studied by $\mathrm{Xu}$ and Bosisio [16]. Bi-anisotropic materials include all the aforementioned materials as particular cases and represent the more general form of linear media. This kind of materials has been studied for circular cross-section guiding structures in $[17,18]$ whereas the completely arbitrary waveguide problem is analysed in [19].

According to [20], any FEM formulation should fit some properties in order to be considered valid for dealing with the waveguide problem. First of all, and probably the most important thing, the formulation should not give non-physical solutions (the so-called spurious modes). Some node-based FEM formulations avoiding the appearance of spurious modes have been reported in the literature (the one presented in [8] has been successfully applied to the analysis of both closed and open lossy dielectric waveguides). Secondly, the nodal FEM formulations hold one important drawback, since they cannot properly represent the singular fields arising in the vicinity of sharp corners [2]. On the contrary, edge-element formulations, as they do not use the value of the field at the corner as an unknown, do not present this problem. On the other hand, a basic division may be established among FEM formulations, by distinguishing between those based on variational principles or Ritz method and those derived via the Galerkin method. The Ritz method has been very used in the past, and is still used even in formulations like those presented in $[4,5,8,11]$. However, when the formulation should be able to deal with fully arbitrary materials, it is not evident to find a functional whose Euler's equation matches the differential equation to be solved. So, when the variational approach is used, only a restricted anisotropy is considered $[4,5]$ or the local potential method is applied to avoid this problem $[8,11]$. In the case of the formulation presented by Pan and Tan in [11], they assume that the propagation constant for waves propagating in the $-z$ direction is the same than that for waves propagating in the $+z$ direction, which is no longer true when the media involved in the analysis are not reciprocal, as it is usual for bi-anisotropic materials. The formulation presented in [10], which is based on a mixed nodal-edge element interpolation, is free of spurious solutions, does not present problems at the corners and is valid for the most general anisotropic materials. However, the eigensystem obtained by the discretization of the wave equations is quadratic, whereas other formulations $[4,5,9,11]$ directly yield generalised eigensystems. Even so, although for solving a quadratic eigensystem it is necessary to transform it into a generalised one by increasing the number of unknowns, the transformation proposed 


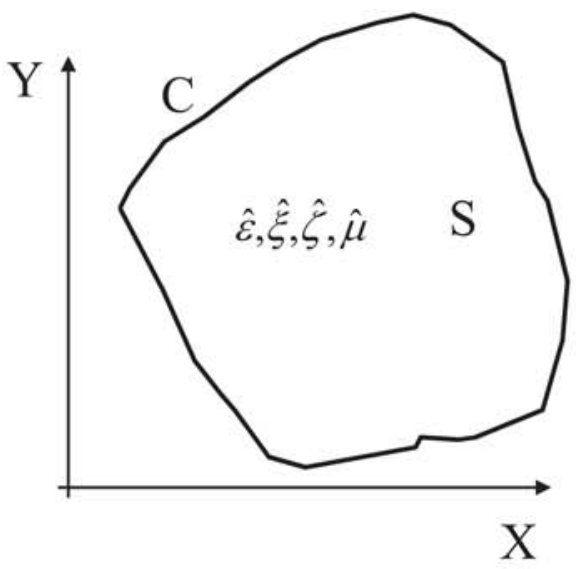

Figure 1: Geometry of the general waveguide problem.

in [10] leads to a number of unknowns (the number of nodes plus twice the number of edges in the FEM mesh) lower than in other similar formulations.

In this paper, the formulation presented in [10] is extended to the study of bi-anisotropic waveguides. In section 2, the wave equation is derived from Maxwell's Equations in general bi-anisotropic waveguides. In section 3, a pair of dual integral equations (for the electric and magnetic fields) is derived from the wave equation using the Galerkin Method. Then, these fields are interpolated using first order edge elements for their transverse components, while the axial components are interpolated using first order Lagrange elements. The discretization of the wake formulation of the wave equation leads to a quadratic sparse eigensystem, which is reduced to a generalised one. In this new eigensystem, the matrices are still sparse and the eigenvalue is closely related to the propagation constant of the modes in the waveguide. Moreover, since edge elements have been used for interpolating the transverse components of the fields, no spurious solutions appear in the eigenvalue spectrum. To show the possibilities of the proposed method, several numerical examples are presented in section 4, comparing (whenever possible) the results obtained by the present method with those reported by other authors. Finally, some conclusions are highlighted.

\section{The wave equation in bianisotropic waveguides}

In this work, we will consider the analysis of the z-invariant waveguide shown in Figure 1, formed by a lossy inhomogeneous and bianisotropic medium enclosed by a perfect conductor (electric or magnetic). The bianisotropic medium will be characterised by four $3 \times 3$ matrices $\left(\hat{\varepsilon}_{r}, \hat{\xi}, \hat{\zeta}\right.$, and $\left.\hat{\mu}_{r}\right)$, and the fields inside it must satisfy the general constitutive relationships:

$$
\begin{aligned}
\vec{D} & =\varepsilon_{0} \hat{\varepsilon}_{r} \vec{E}+\hat{\xi} \vec{H}, \\
\vec{B} & =\mu_{0} \hat{\mu}_{r} \vec{H}+\hat{\zeta} \vec{E},
\end{aligned}
$$

To simplify the analysis, it is convenient to write all the tensors by distinguishing between transversal and axial components

$$
\hat{\varepsilon}_{r}=\left[\begin{array}{ll}
\varepsilon_{t t} & \varepsilon_{t z} \\
\varepsilon_{z t} & \varepsilon_{z z}
\end{array}\right], \quad \hat{\xi}=\left[\begin{array}{ll}
\xi_{t t} & \xi_{t z} \\
\xi_{z t} & \xi_{z z}
\end{array}\right], \quad \hat{\zeta}=\left[\begin{array}{ll}
\zeta_{t t} & \zeta_{t z} \\
\zeta_{z t} & \zeta_{z z}
\end{array}\right], \quad \hat{\mu}_{r}=\left[\begin{array}{ll}
\mu_{t t} & \mu_{t z} \\
\mu_{z t} & \mu_{z z}
\end{array}\right] .
$$

where subscript $t$ refers to the two transverse components $(x, y)$ and $z$ to the axial one. Hence, sub-index $t t$ stands for $2 \times 2$ sub-matrices, $t z$ and $z t$ stand for $2 \times 1$ and $1 \times 2$ vectors, respectively, and $z z$ stands for scalar entries.

Within a general bi-anisotropic waveguide, the source-free Maxwell's equations for time-harmonic fields given by:

$$
\nabla \times \vec{E}=-j \omega \vec{B}, \quad \nabla \times \vec{H}=j \omega \vec{D},
$$

can be applied. 
An exponential dependence of the fields with the $z$ co-ordinate may be assumed, because the geometry of waveguides is invariant with this variable:

$$
\begin{array}{ll}
\vec{E}=\vec{E}_{0}(x, y) e^{-\gamma z}, & \vec{D}=\vec{D}_{0}(x, y) e^{-\gamma z}, \\
\vec{B}=\vec{B}_{0}(x, y) e^{-\gamma z}, & \vec{H}=\vec{H}_{0}(x, y) e^{-\gamma z},
\end{array}
$$

where $\gamma=\alpha+j \beta$ is the complex propagation constant, $\alpha$ being the attenuation constant and $\beta$ the phase constant.

Due to the waveguide axial symmetry, the nabla operator can be split into transverse and axial components:

$$
\nabla=\nabla_{t}+\frac{\partial}{\partial z} \hat{z}
$$

where $\nabla_{t}$ is the part of the nabla operator acting on the transversal coordinates (e.g., $\nabla_{t}=\frac{\partial}{\partial x} \hat{x}+\frac{\partial}{\partial y} \hat{y}$ in rectangular coordinates).

Applying the nabla operator to any field of the form $\vec{A}=\overrightarrow{A_{0}}(x, y) e^{-\gamma z}$ leads to:

$$
\nabla \times \vec{A}=-\gamma e^{-\gamma z} \hat{z} \times \overrightarrow{A_{0}}(x, y)+e^{-\gamma z} \nabla_{t} \times \overrightarrow{A_{0}}(x, y),
$$

Re-ordering equation (11) and introducing the operator $\nabla_{-}=\nabla_{t}-\gamma \hat{z}$ yields

$$
\nabla \times \vec{A}=\nabla_{-} \times \overrightarrow{A_{0}}(x, y) e^{-\gamma z} .
$$

Hence, equations (8) can be re-written as:

$$
\nabla_{-} \times \vec{E}_{0}=-j \omega \vec{B}_{0}, \quad \nabla_{-} \times \vec{H}_{0}=j \omega \vec{D}_{0},
$$

or, introducing the constitutive relationships (6)

$$
\begin{gathered}
\nabla_{-} \times \vec{E}_{0}=-j \omega\left(\mu_{0} \hat{\mu}_{r} \vec{H}_{0}+\hat{\zeta} \vec{E}_{0}\right), \\
\nabla_{-} \times \vec{H}_{0}=j \omega\left(\varepsilon_{0} \hat{\varepsilon}_{r} \vec{E}_{0}+\hat{\xi} \vec{H}_{0}\right) .
\end{gathered}
$$

Solving for $\vec{H}_{0}$ in equation (14a) and introducing it into equation (14b) leads, after some elementary manipulations, to the form:

$$
\left(\nabla_{-} \times-j \omega \hat{\xi}\right) \hat{\mu}_{r}^{-1}\left(\nabla_{-} \times+j \omega \hat{\zeta}\right) \vec{E}_{0}=\omega^{2} c_{0}^{-2} \hat{\varepsilon}_{r} \vec{E}_{0},
$$

where $c_{0}=1 / \sqrt{\varepsilon_{0} \mu_{0}}$ is the speed of light in vacuum.

In a similar way, solving for $\vec{E}_{0}$ in equation (14b) and introducing it into equation (14a) leads to:

$$
\left(\nabla_{-} \times+j \omega \hat{\zeta}\right) \hat{\varepsilon}_{r}^{-1}\left(\nabla_{-} \times-j \omega \hat{\xi}\right) \vec{H}_{0}=\omega^{2} c_{0}^{-2} \hat{\mu}_{r} \vec{H}_{0}
$$

Equations (15) and (16) are the most general form of the wave equation in linear media and their solution, subject to the appropriate boundary conditions on electric $\left(C_{E}\right)$ or magnetic $\left(C_{M}\right)$ walls

$$
\begin{aligned}
& \hat{n} \times\left.\vec{E}_{0}\right|_{C_{E}}=0, \\
& \hat{n} \times\left.\vec{H}_{0}\right|_{C_{M}}=0,
\end{aligned}
$$

provides the propagation modes in the waveguide.

\section{Finite Element solution}

To solve the waveguide problem by using the FEM, it is necessary to derive an integral equation equivalent to the vector wave equation, which some authors have obtained by using the local potential method $[8,11]$. Nevertheless, the weighted residuals method will be applied here for obtaining the integral equations equivalent to (15) and (16). 
Considering equation (15), it is first multiplied by a weighting function $\vec{F}_{0}$ satisfying the same boundary conditions than the unknown $\vec{E}_{0}$ and then integrated on the wave cross-section $S$ :

$$
\iint_{S} \vec{F}_{0} \cdot\left(\nabla_{-} \times-j \omega \hat{\xi}\right) \hat{\mu}_{r}^{-1}\left(\nabla_{-} \times+j \omega \hat{\zeta}\right) \vec{E}_{0} d S=\omega^{2} c_{0}^{-2} \iint_{S} \vec{F}_{0} \cdot \hat{\varepsilon}_{r} \vec{E}_{0} d S,
$$

To reduce the order of the differential operator applied to $\vec{E}_{0}$ in (18) the vector identity

$$
\nabla_{-} \cdot\left(\overrightarrow{A_{0}} \times \vec{F}_{0}\right)=\vec{F}_{0} \cdot\left(\nabla_{-} \times \vec{A}_{0}\right)-\vec{A}_{0} \cdot\left(\nabla_{-} \times \vec{F}_{0}\right)+\gamma \hat{z} \cdot\left(\overrightarrow{A_{0}} \times \vec{F}_{0}\right)
$$

is considered, where the auxiliary vector

$$
\overrightarrow{A_{0}}=\hat{\mu}_{r}^{-1}\left(\nabla_{-} \times+j \omega \hat{\zeta}\right) \vec{E}_{0}
$$

has been defined in order to simplify the notation. Integrating (19) on the the wave cross-section $S$ leads to

$$
\iint_{S} \nabla_{-} \cdot\left(\overrightarrow{A_{0}} \times \vec{F}_{0}\right) d S=\iint_{S} \vec{F}_{0} \cdot\left(\nabla_{-} \times \vec{A}_{0}\right) d S-\iint_{S} \vec{A}_{0} \cdot\left(\nabla_{-} \times \vec{F}_{0}\right) d S+\iint_{S} \gamma \hat{z} \cdot\left(\vec{A}_{0} \times \vec{F}_{0}\right) d S .
$$

Applying a corollary of the divergence theorem to the left hand side of equation (21) leads to:

$$
\iint_{S} \nabla_{-} \cdot\left(\vec{A}_{0} \times \vec{F}_{0}\right) d S=\oint_{C}\left(\vec{F}_{0} \times \vec{A}_{0}\right) \cdot \hat{n} d L-\iint_{S} \gamma \hat{z} \cdot\left(\overrightarrow{A_{0}} \times \vec{F}_{0}\right) d S,
$$

$C$ being the waveguide boundary and $\hat{n}$ the unit vector normal to the waveguide wall pointing outwards.

Assuming that $C=C_{E} \cup C_{M}$, the boundary conditions defined by equations (17) will force the line integral to vanish as far as

$$
\oint_{C}\left(\vec{F}_{0} \times \vec{A}_{0}\right) \cdot \hat{n} d L=\oint_{C_{M}}\left(\hat{n} \times \vec{A}_{0}\right) \cdot \vec{F}_{0} d L-\oint_{C_{E}}\left(\hat{n} \times \vec{F}_{0}\right) \cdot \vec{A}_{0} d L
$$

where the integral along $C_{E}$ vanishes because $\vec{F}_{0}$ must satisfy the boundary condition (17a) while, according to (14a), $\vec{A}_{0}=-j \omega \mu_{0} \vec{H}_{0}$, and therefore the integral along $C_{M}$ also reduces to zero because $\vec{H}_{0}$ must satisfy (17b) on the magnetic wall.

Hence, introducing (22) into (21), the following expression is obtained:

$$
\iint_{S} \vec{F}_{0} \cdot\left(\nabla_{-} \times \vec{A}_{0}\right) d S=\iint_{S} \vec{A}_{0} \cdot\left(\nabla_{-} \times \vec{F}_{0}\right) d S-2 \iint_{S} \gamma \hat{z} \cdot\left(\overrightarrow{A_{0}} \times \vec{F}_{0}\right) d S .
$$

Using the auxiliary vector $\overrightarrow{A_{0}}$, the left hand side of equation (18) can be re-written as:

$$
\iint_{S} \vec{F}_{0} \cdot\left(\nabla_{-} \times-j \omega \hat{\xi}\right) \hat{\mu}_{r}^{-1}\left(\nabla_{-} \times+j \omega \hat{\zeta}\right) \vec{E}_{0} d S=\iint_{S} \vec{F}_{0} \cdot\left(\nabla_{-} \times \vec{A}_{0}\right) d S-j \omega \iint_{S} \vec{F}_{0} \hat{\xi} \vec{A}_{0} d S
$$

Introducing now equation (24) into (25) yields

$$
\iint_{S} \vec{F}_{0} \cdot\left(\nabla_{-} \times-j \omega \hat{\xi}\right) \hat{\mu}_{r}^{-1}\left(\nabla_{-} \times+j \omega \hat{\zeta}\right) \vec{E}_{0} d S=\underbrace{\iint_{S} \vec{A}_{0} \cdot\left(\nabla_{-} \times \vec{F}_{0}\right) d S-2 \iint_{S} \gamma \hat{z} \cdot\left(\vec{A}_{0} \times \vec{F}_{0}\right) d S}_{(a)}-j \omega \iint_{S} \vec{F}_{0} \hat{\xi} \vec{A}_{0} d S
$$

where (a) can be re-arranged as:

$$
\iint_{S} \vec{A}_{0} \cdot\left(\nabla_{-}+2 \gamma \hat{z}\right) \times \vec{F}_{0} d S=\iint_{S} \vec{A}_{0} \cdot\left(\nabla_{+} \times \vec{F}_{0}\right) d S
$$

being $\nabla_{+}=\nabla_{t}+\gamma \hat{z}$.

Introducing now equation (27) into equation (26) leads to:

$$
\iint_{S} \vec{F}_{0} \cdot\left(\nabla_{-} \times-j \omega \hat{\xi}\right) \hat{\mu}_{r}^{-1}\left(\nabla_{-} \times+j \omega \hat{\zeta}\right) \vec{E}_{0} d S=\iint_{S}\left(\nabla_{+} \times \vec{F}_{0}-j \omega \vec{F}_{0} \hat{\xi}\right) \cdot \vec{A}_{0} d S
$$


Finally, introducing equation (28) into equation (18) and taking into account equation (20), the following weak formulation of equation (15) can be obtained:

$$
\iint_{S}\left(\nabla_{+} \times \vec{F}_{0}-j \omega \vec{F}_{0} \hat{\xi}\right) \hat{\mu}_{r}^{-1}\left(\nabla_{-} \times \vec{E}_{0}+j \omega \hat{\zeta} \vec{E}_{0}\right) d S=\omega^{2} c_{0}^{-2} \iint_{S} \vec{F}_{0} \cdot \hat{\varepsilon}_{r} \vec{E}_{0} d S
$$

Following a similar procedure with equation (16) leads to the corresponding weak formulation

$$
\iint_{S}\left(\nabla_{+} \times \vec{G}_{0}+j \omega \vec{G}_{0} \hat{\zeta}\right) \hat{\varepsilon}_{r}^{-1}\left(\nabla_{-} \times \vec{H}_{0}-j \omega \hat{\xi} \vec{H}_{0}\right) d S=\omega^{2} c_{0}^{-2} \iint_{S} \vec{G}_{0} \hat{\mu}_{r} \vec{H}_{0} d S,
$$

$\vec{G}_{0}$ being the test functions, which satisfy the same boundary conditions as $\vec{H}_{0}$.

By using the differential operators $\nabla_{-}$and $\nabla_{+}$very compact expressions for these integral equations can be derived and, because of the dual form of equations (29)- (30), only the finite element scheme for the electric field, corresponding to equation (29), will be presented.

A fixed (i.e., without adaptive refinement) 3-simplex mesh is defined on the solution domain $S$ and, following the procedure described in [21], the transverse components of $\vec{E}_{0}$ and $\vec{F}_{0}\left(\vec{E}_{0 t}\right.$ and $\left.\vec{F}_{0 t}\right)$ are interpolated at the mesh edges using Whitney 1-forms $W^{1}$ whilst Whitney 0 -forms $W^{0}$ are used to interpolate the corresponding axial components $E_{0 z}$ and $F_{0 z}$ at the mesh nodes, leading to:

$$
\vec{E}_{0}=\left[\begin{array}{cc}
P_{t t}^{T} & 0 \\
0 & P_{z z}^{T}
\end{array}\right]\left[\begin{array}{c}
\dot{E}_{t} \\
\dot{E}_{z}
\end{array}\right], \quad \vec{F}_{0}=\left[\begin{array}{cc}
P_{t t}^{T} & 0 \\
0 & P_{z z}^{T}
\end{array}\right]\left[\begin{array}{c}
\dot{F}_{t} \\
\dot{F}_{z}
\end{array}\right], \quad P_{t t}=\left[\begin{array}{ll}
U & V
\end{array}\right], \quad P_{z z}=[N],
$$

where the dots over the unknown and the weighting functions stand for their corresponding values at edges or nodes.

In (31) the elements of matrix $N$ are the Whitney 0 -forms used to interpolate $E_{0 z}$ and $F_{0 z}$ :

$$
N_{i}=\sum_{e=1}^{N_{e}} \psi_{i}^{e}(x, y)
$$

$\psi_{i}^{e}(x, y)$ being the first order Lagrange interpolating function for the i-th mesh node within the e-th triangle and $N_{e}$ the number of mesh elements.

The Whitney 1-forms used to interpolate $\vec{E}_{0 t}$ and $\vec{F}_{0 t}$ at the mesh edges are vector functions of the form:

$$
\vec{N}_{i}(x, y)=L_{i} \sum_{e=1}^{N_{e}}\left[\psi_{i-}(x, y) \nabla \psi_{i+}(x, y)-\psi_{i+}(x, y) \nabla \psi_{i-}(x, y)\right]=U_{i} \hat{x}+V_{i} \hat{y},
$$

where $L_{i}$ is the length of the i-th edge in the mesh and the subscripts - and + stand for its origin and end nodes, respectively. Elements of vectors $U$ and $V$ of sub-matrix $P_{t t}$ in (31) are then defined by (33).

After the Whitney form interpolation, the differential operations involved in (29) give

$$
\nabla_{-} \times \vec{E}_{0}=\left[\begin{array}{cc}
-\gamma Q_{t t}^{T} & Q_{z t}^{T} \\
Q_{t z}^{T} & 0
\end{array}\right]\left[\begin{array}{c}
\dot{E}_{t} \\
\dot{E}_{z}
\end{array}\right], \quad \nabla_{+} \times \vec{F}_{0}=\left[\begin{array}{cc}
\gamma Q_{t t}^{T} & Q_{z t}^{T} \\
Q_{t z}^{T} & 0
\end{array}\right]\left[\begin{array}{c}
\dot{F}_{t} \\
\dot{F}_{z}
\end{array}\right],
$$

where

$$
Q_{t t}=\left[\begin{array}{ll}
-V & U
\end{array}\right], \quad Q_{t z}=\left[\begin{array}{ll}
V_{x}-U_{y}
\end{array}\right], \quad Q_{z t}=\left[\begin{array}{ll}
N_{y} & -N_{x}
\end{array}\right],
$$

the subindices $x$ and $y$ in the interpolating functions standing for differentiation with respect to the corresponding variable.

After some algebraic manipulations, the following equation is obtained

$$
\gamma^{2}\left[\dot{F}_{t}^{T} \dot{F}_{z}^{T}\right]\left[\begin{array}{cc}
A_{t t} & 0 \\
0 & 0
\end{array}\right]\left[\begin{array}{c}
\dot{E}_{t} \\
\dot{E}_{z}
\end{array}\right]+\gamma\left[\dot{F}_{t}^{T} \dot{F}_{z}^{T}\right]\left[\begin{array}{cc}
B_{t t} & B_{t z} \\
B_{z t} & 0
\end{array}\right]\left[\begin{array}{c}
\dot{E}_{t} \\
\dot{E}_{z}
\end{array}\right]+\left[\begin{array}{l}
\dot{F}_{t}^{T} \dot{F}_{z}^{T}
\end{array}\right]\left[\begin{array}{cc}
C_{t t} & C_{t z} \\
C_{z t} & C_{z z}
\end{array}\right]\left[\begin{array}{c}
\dot{E}_{t} \\
\dot{E}_{z}
\end{array}\right]=0
$$


where

$$
\begin{aligned}
A_{t t}= & -\iint_{S} Q_{t t} v_{t t} Q_{t t}^{T} d S, \\
B_{t t}= & \iint_{S}\left[Q_{t t}\left(v_{t z}-v_{z t}^{T}\right) Q_{t z}^{T}+j \omega P_{t t}\left(\xi_{t t} v_{t t}+\zeta_{t t}^{T} v_{t t}^{T}+\xi_{t z} v_{z t}+\zeta_{z t}^{T} v_{t z}^{T}\right) Q_{t t}^{T}\right] d S, \\
B_{t z}= & \iint_{S}\left[Q_{t t} v_{t t} Q_{z t}^{T}+j \omega Q_{t t}\left(v_{t t} \zeta_{t z}-v_{t z} \zeta_{z z}\right) P_{z z}^{T}\right] d S, \\
B_{z t}= & \iint_{S}\left[-Q_{z t} v_{t t} Q_{t t}^{T}+j \omega P_{z z}\left(\xi_{z t} v_{t t}+\xi_{z z} v_{z t}\right) Q_{t t}^{T}\right] d S, \\
C_{t t}= & \iint_{S}\left[Q_{t z} v_{z z} Q_{t z}^{T}+j \omega Q_{t z}\left(v_{z t} \zeta_{t t}+v_{z z} \zeta_{z t}-v_{z z}^{T} \xi_{t z}^{T}-v_{t z}^{T} \xi_{t t}^{T}\right) P_{t t}^{T}+\omega^{2} P_{t t}\left(\xi_{t t} v_{t t} \zeta_{t t}+\xi_{t z} v_{z t} \zeta_{t t}-c_{0}^{2} \varepsilon_{t t}\right) P_{t t}^{T}\right] d S, \\
C_{t z}= & \iint_{S}\left[Q_{t z} v_{z t} Q_{z t}^{T}+j \omega Q_{t z}\left(v_{z t} \zeta_{t z}+v_{z z} \zeta_{z z}\right) P_{z z}^{T}-P_{t t}\left(\xi_{t t} v_{t t}-\xi_{t z} v_{z t}\right) Q_{z t}^{T}\right. \\
& \left.+\omega^{2} P_{t t}\left(\xi_{t t} v_{t t} \zeta_{t z}+\xi_{t z} v_{z t} \zeta_{t z}+\xi_{t t} v_{t z} \zeta_{z z}+\xi_{t z} v_{z z} \zeta_{z z}-c_{0}^{2} \varepsilon_{t z}\right) P_{z z}^{T}\right] d S, \\
C_{z t}= & \iint_{S}\left[Q_{z t} v_{t z} Q_{t z}^{T}+j \omega Q_{z t}\left(v_{t t} \zeta_{t t}+v_{t z} \zeta_{z t}\right) P_{t t}^{T}-j \omega P_{z z}\left(\xi_{z t} v_{t z}+\xi_{z z} v_{z z}\right) Q_{t z}^{T}\right. \\
& \left.+\omega^{2} P_{z z}\left(\xi_{z t} v_{t t} \zeta_{t t}+\xi_{z z} v_{z t} \zeta_{t t}+\xi_{z t} v_{t z} \zeta_{z t}+\xi_{z z} v_{z z} \zeta_{z t}-c_{0}^{2} \varepsilon_{z t}\right) P_{t t}^{T}\right] d S, \\
C_{z z}= & \iint_{S}\left[Q_{z t} v_{t t} Q_{z t}^{T}+j \omega Q_{z t}\left(v_{t t} \zeta_{t z}+v_{t z} \zeta_{z z}\right) P_{z z}^{T}-j \omega P_{z z}\left(\xi_{z t} v_{t t}+\xi_{z z} v_{z t}\right) Q_{z t}^{T}\right. \\
& \left.+\omega^{2} P_{z z}\left(\xi_{z t} v_{t t} \zeta_{t z}+\xi_{z z} v_{z t} \zeta_{t z}+\xi_{z t} v_{t} \zeta_{z z}+\xi_{z z} v_{z z} \zeta_{z z}-c_{0}^{2} \varepsilon_{z z}\right) P_{z z}^{T}\right] d S,
\end{aligned}
$$

$v$ standing for $\hat{\mu}_{r}^{-1}$.

According to the weighted residuals method, as far as equation (36) must be valid for any test function $\vec{F}_{0}$ in the solutions space it shall also be valid for any of the basis functions used in the interpolation. Therefore, equation (36) reduces to the following quadratic eigensystem:

$$
\gamma^{2}\left[\begin{array}{cc}
A_{t t} & 0 \\
0 & 0
\end{array}\right]\left[\begin{array}{l}
\dot{E}_{t} \\
\dot{E}_{z}
\end{array}\right]+\gamma\left[\begin{array}{cc}
B_{t t} & B_{t z} \\
B_{z t} & 0
\end{array}\right]\left[\begin{array}{l}
\dot{E}_{t} \\
\dot{E}_{z}
\end{array}\right]+\left[\begin{array}{cc}
C_{t t} & C_{t z} \\
C_{z t} & C_{z z}
\end{array}\right]\left[\begin{array}{l}
\dot{E}_{t} \\
\dot{E}_{z}
\end{array}\right]=0 .
$$

It is worth to point out that the quadratic eigensystem (38) is completely free of spurious solutions and the matrices involved are highly sparse, thus allowing a great memory and time saving.

The transformation of (38) into a generalised sparse eigensystem can be achieved now by introducing $\lambda=1 / \gamma$ and $\bar{E}_{t}=\dot{E}_{t} / \lambda$, thus duplicating the number of transversal unknowns. The resulting equation has the form

$$
\lambda M \tilde{E}=N \tilde{E},
$$

where

$$
M=\left[\begin{array}{ccc}
C_{t t} & C_{t z} & 0 \\
C_{z t} & C_{z z} & 0 \\
0 & 0 & I
\end{array}\right], \quad N=\left[\begin{array}{ccc}
-B_{t t} & -B_{t z} & -A_{t t} \\
-B_{z t} & 0 & 0 \\
I & 0 & 0
\end{array}\right], \quad \tilde{E}=\left[\begin{array}{c}
\dot{E}_{t} \\
\dot{E}_{z} \\
\bar{E}_{t}
\end{array}\right] .
$$

The boundary condition set by equation (17a) is applied to equation (39) by removing rows and columns corresponding to the simplex nodes and edges laying on perfect electric conducting surfaces, leading to:

$$
\lambda M^{0} \tilde{E}^{0}=N^{0} \tilde{E}^{0},
$$

The dimension of $M^{0}$ null-space is given by the number of free nodes (i.e., those non lying on the domain boundary) in the simplex mesh and therefore there will be as many eigenvalues $\lambda=0$ as elements has the vector $\dot{E}_{z}^{0}$, which correspond to

$$
\dot{E}_{t}^{0}=0, \bar{E}_{t}^{0}=-\left(A_{t t}^{0}\right)^{-1},|\gamma|=\infty .
$$

These non-valid solutions are perfectly defined and located, an they must not be confused with any kind of spurious modes. The generalized eigenproblem defined by (41) has been solved using the freely-available software library SLEPc [22] with the default settings. 


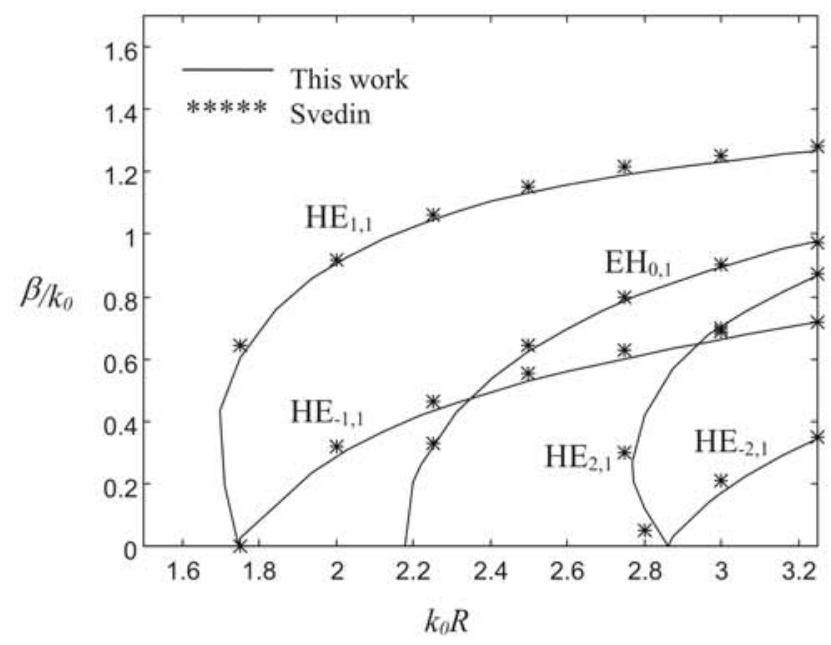

Figure 2: Normalised phase constant for the first five modes in a circular homogeneous chirowaveguide of radius R.

\section{Numerical results}

In this section, several waveguides have been analysed to show the validity and versatility of the proposed FEM formulation. Whenever possible, the computed results have been compared with previously published results. In other cases, very general examples have been solved, and their results are presented in order to be used as a reference in future works.

The first example considered is a circular chirowaveguide of radius $\mathrm{R}$ filled with a non-magnetic $\left(\mu=\mu_{0}\right)$ material characterised by $\varepsilon_{r}=1.1419 \varepsilon_{0}$ and $\xi=-\zeta=j \mu_{0} \zeta_{c}$, with a chirality admittance $\zeta_{c}=1 \mathrm{mS}$. Figure 2 shows the propagation constants for the first $E H_{m n}$ and $H E_{m n}$ modes nomalized with respect to the free space wave-number $k_{0}$. The results are compared with those published by Svedin in [13], showing a very good agreement. Only the phase constant $\beta$ is plotted in Figure 2 because in this case $\alpha=0$. The analysis has been carried out in terms of electric field, yielding a final eigensystem with 450 unknowns.

Next, the normalized propagation constant for the first mode of a rectangular waveguide filled with a non-magnetic chiral material with $\varepsilon=\varepsilon_{0}$ has been computed for different values of the chirality admittance. Figure 3 shows the waveguide dimensions (in terms of the free-space wavelength $\left.\lambda_{0}\right)$ along with the normalized phase constant $\beta(\alpha=0$ for this mode). The results were obtained using the magnetic field formulation yielding a final eigensystem with 520 unknowns. The computed results are also compared in Figure 3 with the values obtained by Xu and Bosisio in reference [16], showing a high similarity between them.

The third example presented in this paper is devoted to show how the formulation works with general bianisotropic materials. To do so, a circular waveguide of radius $\mathrm{R}$ has been analysed, with 1066 unknowns in the final eigensystem using the E-field formulation. The material filling the waveguide has the following constitutive parameters

$$
\begin{gathered}
\hat{\varepsilon}_{r}=\left[\begin{array}{ccc}
1.099 & -j 0.043 & 0 \\
j 0.043 & 1.099 & 0 \\
0 & 0 & 1.142
\end{array}\right], \\
\hat{\mu}_{r}=\left[\begin{array}{ccc}
0.7 & -j 0.3 & 0 \\
j 0.3 & 0.7 & 0 \\
0 & 0 & 1
\end{array}\right],
\end{gathered}
$$

and $\hat{\xi}=-\hat{\zeta}=j \zeta_{c} \mu_{0} \hat{\mu}_{r}$, with a chirality admittance $\zeta_{c}=1 \mathrm{mS}$. The normalized phase constants for the first two $H E_{m n}$ modes are plotted in Figure 4 (as in the two previous examples, $\alpha=0$ ), along with the values presented by Shen in [14], the results being very similar.

The last waveguide analyzed is a very general one: the trapezoidal microstrip line of Figure 5. In this structure, the material 1 has the same constitutive parameters as the chirowaveguide of the first example, material 2 has the 


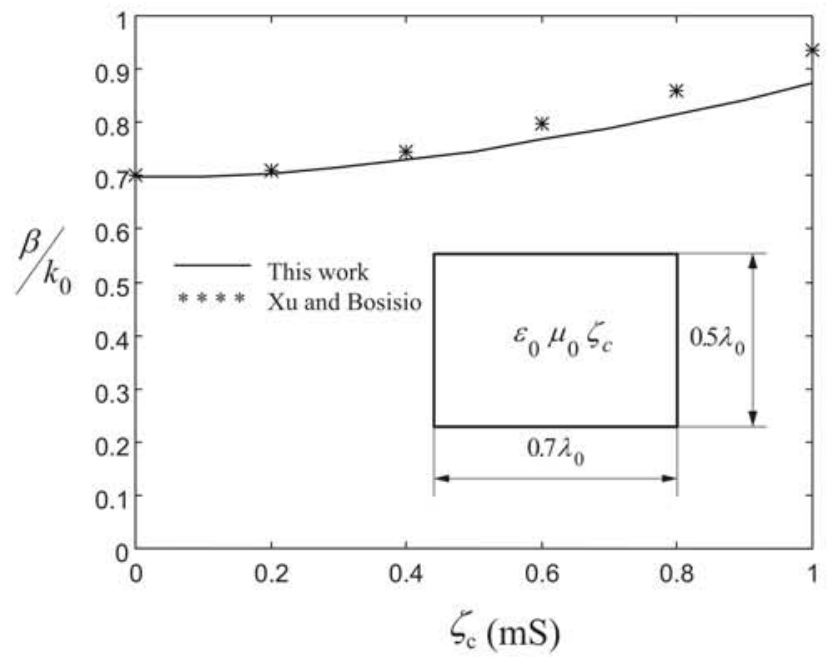

Figure 3: Normalised phase constant for the fundamental mode of a rectangular chirowaveguide, represented versus the chirality admittance $\zeta_{c}$.

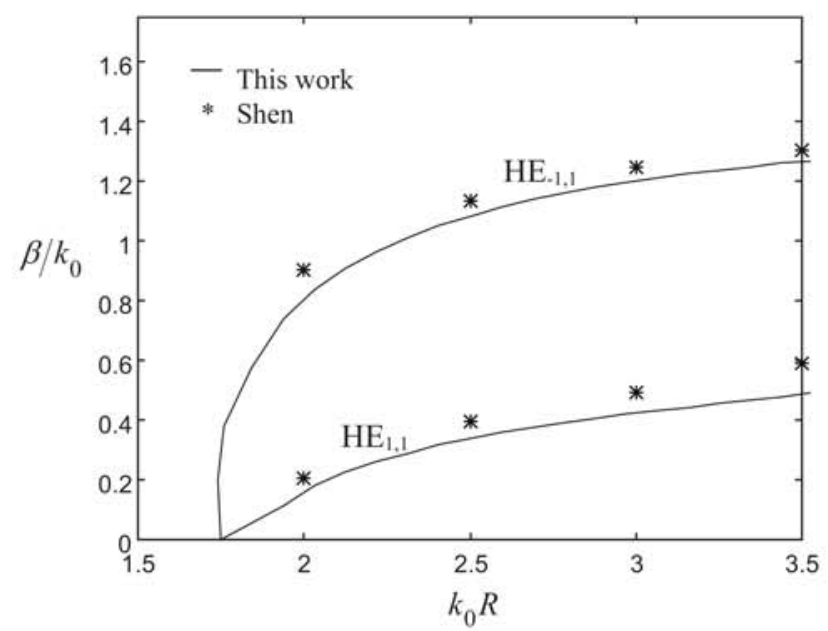

Figure 4: Normalised phase constant for the first two modes in a circular homogeneous gyrotropic chirowaveguide of radius R. 


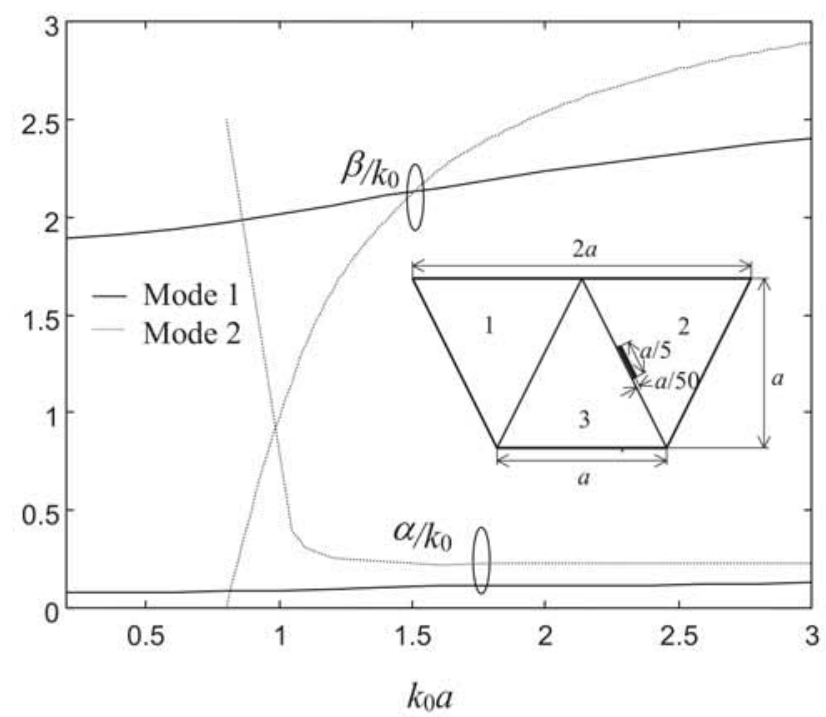

Figure 5: Normalized propagation constant for the first two modes of a bi-anisotropic inhomogeneous microstrip waveguide with trapezoidal cross-section.

same properties as the general bianisotropic circular waveguide of the third example, and material 3 is an anisotropic $(\hat{\xi}=\hat{\zeta}=0)$, non-magnetic dielectric with:

$$
\hat{\varepsilon}_{r}=\left[\begin{array}{ccc}
11.86-j 0.8 & 0 & 0 \\
0 & 20.83-j 3.16 & 0 \\
0 & 0 & 11.86-j 0.8
\end{array}\right] .
$$

This problem has been solved using the E-field formulation, and the final eigensystem involved 700 unknowns. The propagation constant (both $\alpha$ and $\beta$ ) for the first two modes of this structure is also shown in 5. This waveguide has not been previously analysed, and the results are presented here to be used as a reference for future works. Figure 6 shows the transversal electric field for the first two modes of the trapezoidal waveguide.
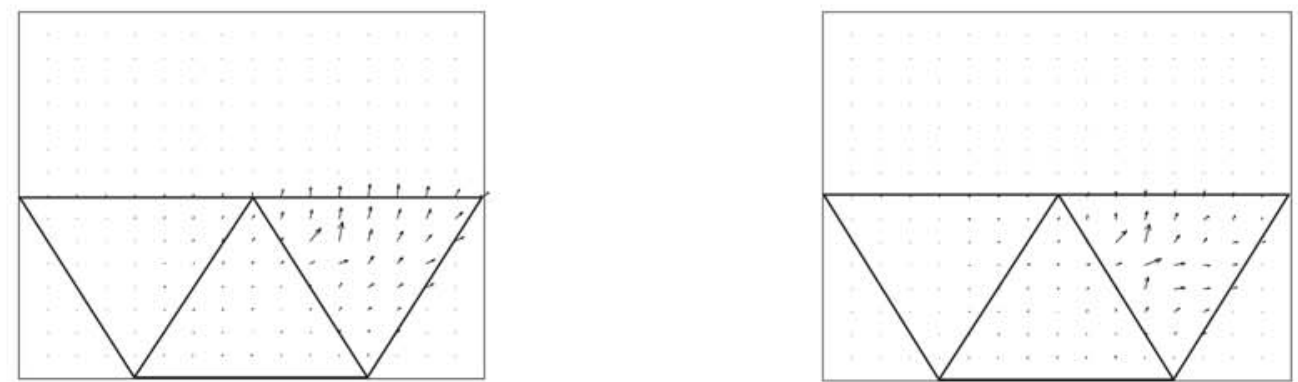

Figure 6: Transversal electric field for the first (left) and second (right) modes in the bi-anisotropic trapezoidal waveguide shown in Figure 5.

\section{Conclusions}

A very powerful FEM formulation based on a Galerkin approach, which is valid for the analysis of most general linear waveguides, has been presented. To prevent the appearance of non-physical solutions, the proposed method uses mixed interpolating functions for the field and the test functions. This strategy yields a spurious free, generalised eigensystem where the eigenvalues are closely related to the propagation constant of the waveguide modes. Several examples have been presented, showing a very good agreement with previously published results. Moreover, a very 
general example is presented in order to be used as reference for future works. Finally, the proposed method has shown to be very efficient, since it leads to a very sparse eigensystem, thus allowing a great reduction in memory requirements and $\mathrm{CPU}$ time.

\section{References}

[1] Elliott, Robert Stratman, An introduction to guided waves and microwave circuits, Prentice-Hall, 1993.

[2] Collin, Robert E, Field theory of guided waves, IEEE Press, 1990.

[3] Dillon, M. B., Gibson, Andrew A. P. and Webb, Jon P., Cut-off and phase constants of partially filled axially magnetized, gyromagnetic waveguides using finite elements, IEEE Transactions on Microwave Theory and Techniques, 41 (5) (1993), 803-808.

[4] Anderson, B. C. and Cendes, Z. J., Solution of ferrite loaded waveguide using vector Finite Elements, IEEE Transactions on Magnetics, 41 (3) (1995), 1578-1581.

[5] Zhou, Lezhu and Davis, Lionel E., Finite-Element method with edge elements for waveguides loaded with ferrite magnetized in arbitrary direction, IEEE Transactions on Microwave Theory and Techniques, 44 (6) (1996) 809-815.

[6] Chen, Yinchao and Beker, Benjamin, Application of MoL to shielded microstrip lines with bi-anisotropic biaxial substrates and cover layers, IEEE Transactions on Magnetics, 30 (5) (1994) 3212-3215.

[7] Polycarpou, Anastasis C., Lyons, Michael R. and Balanis, Constantine A., Finite Element analysis of MMIC waveguide structures with anisotropic substrates, IEEE Transactions on Microwave Theory and Techniques, 44 (10) (1996) 1650-1663.

[8] Lu, Yilong and Fernandez, F. Anibal, An efficient finite element solution of inhomogeneous anisotropic and lossy dielectric waveguides, IEEE Transactions on Microwave Theory and Techniques, 41 (6) (1993) 1215-1223.

[9] Lee, J. F., Finite Element analysis of lossy dielectric waveguides, IEEE Transactions on Microwave Theory and Techniques, 42 (6) (1994) 1025-103.

[10] Nuño, Luis, Balbastre, Juan V. and Castañe, Hector, Analysis of general lossy inhomogeneous and anisotropic waveguides by the finiteelement method (FEM) using edge elements, IEEE Transactions on Microwave Theory and Techniques, 45 (3) (1997) $446-449$.

[11] Pan, G. and Tan, J., General edge element approach to lossy and dispersive structures in anisotropic media, IEE Proceedings-Microwaves, Antennas and Propagation, 144 (2) (1997) 81-90.

[12] Lindell, Ismo V., Sihvola, A. H., Tretyakov, S. A. and Viitanen, A.J., Electromagneic Fields in bi-isotropic media, Artech House, 1994.

[13] Svedin, Jan A. M., Propagation analysis of chirowaveguides using the finite-element method, IEEE Transactions on Microwave Theory and Techniques, 38 (10) (1990) 1488-1496.

[14] Shen, Zhongxiang, The theory of chiroferrite waveguides, Microwave and Optical Technology Letters, 6 (7) (1993) 397-401.

[15] Wenyan, Yin, Wenbing, Wang and Pao, Li, Guided electromagnetic waves in gyrotropic chirowaveguides, IEEE Transactions on Microwave Theory and Techniques, 42 (11) (1994) 2156-2163.

[16] Xu, Yansheng and Bosisio, Renato G., An efficient method for study of general bi-anisotropic waveguides, IEEE Transactions on Microwave Theory and Techniques, 43 (4) (1995) 873-879.

[17] Jakoby, Bernhard and De Zutter, Daniel, Analysis of guided waves in inhomogeneous bianisotropic cylindrical waveguides, IEEE Transactions on Microwave Theory and Techniques, 44 (2) (1996) 297-310.

[18] Graglia, Roberto D., Sarto, Maria S. and Uslenghi, Piergiorgio L.E., TE and TM modes in cylindrical metallic structures filled with bianisotropic material, IEEE Transactions on Microwave Theory and Techniques, 44 (8) (1996) 1470-1477.

[19] Valor, Luis and Zapata, Juan, An efficient finite element formulation to analyze waveguides with lossy inhomogeneous bi-anisotropic materials, IEEE Transactions on Microwave Theory and Techniques, 44 (2) (1996) 291-296.

[20] Fernandez, F. Anibal and Lu, Yilong, Microwave and optical waveguide analysis by the finite element method, John Wiley \& Sons, Inc., 1996.

[21] Lee, Jin-Fa, Sun, D. K. and Cendes, Z. J., Tangential vector finite elements for electromagnetic field computation, IEEE Transactions on Magnetics, 27 (5) (1991) 4032-4035.

[22] Hernández, Vicente, Román, José Enrique, Vidal, Vicente, SLEPc: Scalable Library for Eigenvalue Problem Computations, Lecture Notes in Computer Science, vol. 2565 (2003), p. 377-391. 